\title{
Effect of M2 Macrophages on Injury and Apoptosis of Renal Tubular Epithelial Cells Induced by Calcium Oxalate Crystals
}

\author{
Quan Liu $^{a} \quad$ Yunlong Liu ${ }^{a} \quad$ Xiaofeng Guan ${ }^{a} \quad$ Jihua Wu ${ }^{a} \quad$ Ziqi He \\ Juening Kang ${ }^{a}$ Zhiwei Tao ${ }^{a}$ Yaoliang Deng ${ }^{a, b}$ \\ a Department of Urology, The First Affiliated Hospital of Guangxi Medical University, \\ Nanning, China; ${ }^{b}$ Department of Urology, The Langdong Hospital of Guangxi Medical \\ University, Nanning, China
}

\section{Keywords}

M2 macrophages · Renal tubular epithelial cells · Calcium oxalate crystals · Apoptosis

\begin{abstract}
Background: M2 macrophages have important roles in diseases such as tumours, cardiovascular diseases and renal diseases. This study aimed to determine the effects and protective mechanism of M2 macrophages against oxidative stress injury and apoptosis induced by calcium oxalate crystals ( $\mathrm{CaOx}$ ) in renal tubular epithelial cells (HK-2) under coculture conditions. Methods: THP-1 cells were induced to differentiate into M2 macrophages by using phorbol12-myristate-13-acetate, IL-4 and IL-13. Morphological features were observed by microscopy. Phenotypic markers were identified by reverse transcription-polymerase chain reaction, Western blot and enzyme-linked immunosorbent assay (ELISA). HK-2 cells were treated with $0.5 \mathrm{mg} / \mathrm{mL} \mathrm{CaO}$ crystals and co-cultured with M2 macrophages or apocynin. The viability of HK-2 cells was detected by CCK-8 assay. The lactate dehydrogenase (LDH) activity of HK-2 cells was analysed using a microplate reader. The apoptosis of HK-2 cells was examined by flow cytometry and Hoechst 33258 staining. Reactive oxygen species (ROS) expression and mitochondrial membrane potential in HK-2 cells were detected by a fluorescence microplate reader. Western blot analysis was conducted to detect the expression of p47phox, Bcl-2, cleaved caspase-3, cytochrome c, p38 MAPK, phospho-p38 MAPK, Akt and phospho-Akt. Results: The results of morphology, reverse transcription-polymerase chain reaction, Western blot and ELISA showed that THP-1 cells were successfully polarised to M2 macrophages. The results of co-culture suggested that M2 macrophages or apocynin significantly increased the cell viability and decreased the LDH activity and apoptosis rate after HK-2 cells were challenged with $\mathrm{CaOx}$ crystals. The expression of the p47phox protein and the concentration of ROS were re-
\end{abstract}

Quan Liu and Yunlong Liu contributed equally to this work and therefore share first authorship.

Yaoliang Deng and Zhiwei Tao

Department of Urology

The First Affiliated Hospital of Guangxi Medical University

Nanning 530021, Guangxi Zhuang Autonomous Region (China)

E-Mail dylkf317@163.com and $791562693 @ q q . c o m$ 


\section{Kidney \\ Blood Pressure \\ Research}

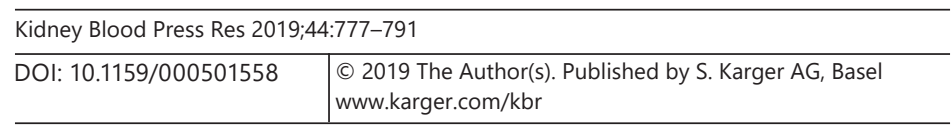

Liu et al.: M2 Macrophages Reduced Apoptosis of HK-2 Cells Induced by CaOx Crystals

duced, the release of mitochondrial membrane potential and the expression of the Bcl-2 protein were upregulated and the protein expression of cleaved caspase- 3 and cytochrome $c$ was downregulated. The expression of the phosphorylated form of p38 MAPK increased. Under coculture conditions with M2 macrophages, the Akt protein of HK-2 cells treated with $\mathrm{CaOx}$ crystals was dephosphorylated, but the phosphorylated form of Akt was not reduced by apocynin. Conclusions: M2 macrophages reduced the oxidative stress injury and apoptosis of HK-2 cells by downregulating the activation of NADPH oxidase, reducing the production of ROS, inhibiting the phosphorylation of p38 MAPK and enhancing the phosphorylation of Akt. We have revealed one of the possible mechanisms by which $\mathrm{M} 2$ macrophages reduce the formation of kidney stones.

(C) 2019 The Author(s)

Published by S. Karger AG, Basel

\section{Introduction}

Kidney stones are a common clinical disease that has an increasing prevalence rate and a recurrence rate of approximately $50 \%$ after 10 years of treatment [1-3]. The prevalence and recurrence rates of this disease are high because the mechanism of the formation of kidney stones remains unclear; kidney stones have epidemiological and histopathological correlations with nephropathy, which may lead to chronic kidney disease and end-stage renal disease [4]. Therefore, kidney stones pose a great threat to human health. Studying the pathogenesis and prevention of the formation of kidney stones is of great significance.

Macrophages exhibit the strongest plasticity in the haematopoietic system and play an important role in development, homeostasis, tissue repair and immunity development; however, these repair and homeostatic functions may be destroyed by chronic damage, indicating a certain relationship between macrophages and diseases [5]. Under different microenvironments in vivo, macrophages can be activated as M1 or M2 macrophages; the former are pro-inflammatory and cause damage, and the latter are anti-inflammatory and promote tissue repair and tumour growth [6]. M1 and M2 macrophages have important roles in diseases such as tumours, cardiovascular diseases and renal diseases [7-9]. The relationship between macrophages and diseases has become a research hotspot.

Calcium oxalate $(\mathrm{CaOx})$ crystals can directly induce macrophages to produce a strong immune response through cell-crystal reactions during the formation of kidney stones. Macrophages migrate around CaOx crystals in the renal interstitium and exert phagocytosis to clear the stone crystals [10]. In addition, different types of macrophages play different roles in the formation of kidney stones: M1 macrophages promote renal stone formation, and M2 macrophages inhibit the formation of kidney stones [11]. For example, renal crystal deposition in CSF-1 gene-deficient mice is more obvious than that in normal wild-type mice [12]. Phagocytic crystals in M2 macrophages are more powerful than M1 macrophages [13]. However, the mechanism through which M2 macrophages inhibit the formation of kidney stones has not yet been fully understood.

Kidney stones are the main part of urolithiasis. The mechanism of their formation includes supersaturation, nucleation, growth, aggregation and retention of kidney stones in tubular cells [14]. CaOx is the main component, accounting for $70-80 \%$ of the organic components of kidney stones [15]. CaOx crystals cause cellular damage, upregulate NADPH oxidase p47phox expression and promote the release of large amounts of reactive oxygen species (ROS) to renal tubular epithelial cells $[16,17]$. ROS are involved in oxidative stress injury in renal tubular epithelial cells [18]. ROS can damage the mitochondrial membrane and reduce its transmembrane potential, causing apoptosis [19]. When crystals induce the apoptosis of renal tubular epithelial cells, the adhesion of crystal stones significantly increases, thereby 


\section{Kidney \\ Blood Pressure \\ Research}

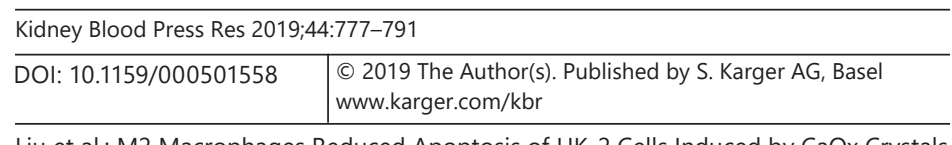

Liu et al.: M2 Macrophages Reduced Apoptosis of HK-2 Cells Induced by CaOx Crystals

promoting the formation of kidney stones [20]. Therefore, CaOx crystal-induced oxidative stress injury and apoptosis in renal tubular epithelial cells play a very important role in the formation of kidney stones.

Apocynin, a constituent of the Himalayan herb Picrorhiza kurroa Royle, has been used as an inhibitor of NADPH oxidase. In addition, researchers have confirmed that Apocynin can inhibit cellular oxidative stress damage and apoptosis $[21,22]$. However, the effect of M2 macrophages on the injury and apoptosis of renal tubular epithelial cells induced by $\mathrm{CaOx}$ crystals during kidney stone formation remains unclear. Therefore, this study used THP-1 cells to polarise M2 macrophages and used apocynin as a positive-control treatment. Co-culture experiments were conducted to analyse and investigate the effects of M2 macrophages on the oxidative stress injury and apoptosis of HK-2 cells induced by $\mathrm{CaOx}$ crystals.

\section{Materials and Methods}

\section{Cell Culture and Macrophage Polarisation}

THP-1 cells (Cell Bank of the Chinese Academy of Sciences, Shanghai, China) were cultured in RPMI-1640 (Gibco, Grand Island, NE, USA) conditioned medium containing 10\% foetal bovine serum (Lonsera, Lonsa Science SRL, Uruguay) and 1\% antibiotics. HK-2 cells (Cell Bank of the Chinese Academy of Sciences, Shanghai, China) were cultured in DMEM/F12 (Gibco, Grand Island, NE, USA) conditioned medium containing 10\% foetal bovine serum and $1 \%$ antibiotics. The cells were cultured in an incubator at $37^{\circ} \mathrm{C}$ and $5 \% \mathrm{CO}_{2}$. THP- 1 cells in the logarithmic growth phase were adjusted to a density of $2 \times 10^{5} / \mathrm{mL}$ and induced with 10 $\mathrm{ng} / \mathrm{mL}$ phorbol-12-myristate-13-acetate (Sigma-Aldrich, USA) for $24 \mathrm{~h}$ to differentiate into M0 macrophages. The cell supernatant was removed, washed 3 times with PBS and added to RPMI-1640 conditioned medium containing $100 \mathrm{ng} / \mathrm{mL}$ LPS (Sigma-Aldrich, USA) and $10 \mathrm{ng} /$ mL IFN- $\gamma$ (Pepro Tech, USA) for $48 \mathrm{~h}$ to polarise M1 macrophages or to the medium containing $20 \mathrm{ng} / \mathrm{mL}$ interleukin (IL)-4 (PeproTech, USA) and $20 \mathrm{ng} / \mathrm{mL}$ IL-13 (PeproTech, USA) for 48 $\mathrm{h}$ to polarise M2 macrophages. The control group M0 was cultured in RPMI-1640 conditioned medium for $48 \mathrm{~h}$. After the macrophages were polarised, they were photographed under a fluorescence microscope (Olympus, Tokyo, Japan) at 100× magnification.

\section{Establishment of a Cocultivation Model}

Following the M2 macrophage induction method described earlier, THP-1 cells were seeded in Transwell upper chambers $(0.4 \mu \mathrm{m}$, Corning, USA) and polarised to M2 macrophages. The supernatant was then aspirated, the cells were washed 3 times with PBS and the medium was replaced with $1 \mathrm{~mL}$ of RPMI-1640 medium. Apocynin was diluted to $50 \mathrm{ng} / \mathrm{mL}$ in RPMI-1640 medium and added to the upper chamber. CaOx was diluted to $0.5 \mathrm{mg} / \mathrm{mL}$ in DMEM/F12 medium and added to HK-2 cells in the lower chamber.

\section{Reverse Transcription-Polymerase Chain Reaction}

After the macrophages were polarised, total RNA was extracted according to the RNAiso Plus instructions (TaKaRa, Japan) and measured. Total RNA ( $1 \mu \mathrm{g})$ was used for reverse transcription into cDNA. The reverse transcription conditions were as follows: $37^{\circ} \mathrm{C}$ for $15 \mathrm{~min}$ and $85^{\circ} \mathrm{C}$ for $5 \mathrm{~s}$. The amplification conditions were as follows: 40 cycles of $95^{\circ} \mathrm{C}$ for $30 \mathrm{~s}$, $95^{\circ} \mathrm{C}$ for $5 \mathrm{~s}$, and $60^{\circ} \mathrm{C}$ for $34 \mathrm{~s}$. The primers were designed and synthesised by Shanghai Shenggong Biology Company. The human GAPDH gene was used as an internal reference. The results were analysed using the $2^{-\Delta \Delta C T}$ method. The primer sequences are shown in Table 1. 


\section{Kidney \\ Blood Pressure \\ Research}

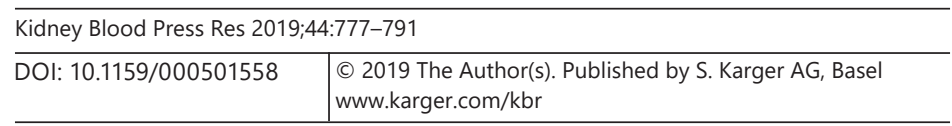

Liu et al.: M2 Macrophages Reduced Apoptosis of HK-2 Cells Induced by CaOx Crystals

Table 1. The sequences of the primers

\begin{tabular}{lll}
\hline Gene name & Forward primer $5^{\prime} \rightarrow 3^{\prime}$ & Reverse primer $5^{\prime} \rightarrow 3^{\prime}$ \\
\hline GAPDH & GTCAGCCGCATCTTCTTT & CGCCCAATACGACCAAAT \\
TNF- $\alpha$ & CCTCAACCTCTTCTGGCTCAA & GTGCAGGCCACACATTCCT \\
IL-1 $\beta$ & TCAGCCAATCTTCATTGCTCAA & TGGCGAGCTCAGGTACTTCTG \\
CD206 & CCGTATGCCGGTCACTGTTA & CAATTCCTCGATGGTGTGGA \\
IL-1ra & TTCATCCGCTCAGACAGTGG & ACGCCTTCGTCAGGCATATT \\
\hline
\end{tabular}

Enzyme-Linked Immunosorbent Assay

After the polarisation of THP-1 cells, the supernatant of each group of cells was collected. IL-6 (CUSABIO, Wuhan, China) and IL-10 (eBioscience, Austria) were detected according to the procedure of the enzyme-linked immunosorbent assay (ELISA) kit. Absorbance was recorded at a wavelength of $450 \mathrm{~nm}$ using a microplate reader (Thermo Scientific, USA). The expression levels of IL- 6 and IL-10 were calculated according to the standard curve.

\section{Cell Viability Assay by CCK-8}

Each group of co-cultured lower-chamber HK-2 cells had the supernatant discarded and was washed with PBS once. The CCK-8 reagent (Dojindo, Kamimashikigun, Kumamoto, Japan) was digested in serum-free medium at a ratio of 1:10 according to the manufacturer's instructions. Each group of cells then had $1.0 \mathrm{~mL}$ of the diluted CCK-8 reagent added, followed by incubation at $37^{\circ} \mathrm{C}$ for $2 \mathrm{~h}$. After incubation, absorbance was recorded at a wavelength of 450 $\mathrm{nm}$ by using a microplate reader. The viability of HK-2 cells in each group was evaluated.

\section{Lactate Dehydrogenase Assay}

The supernatant of each group of HK-2 cells was collected and sequentially added to a 96-well plate according to the instructions of the lactate dehydrogenase (LDH) kit (Nanjing Jiancheng Bioengineering Institute, Nanjing, China). After the sample was added and incubated, absorbance was recorded at $450 \mathrm{~nm}$ wavelength by using a microplate reader. The LDH activity of each group of HK-2 cells was calculated.

\section{Hoechst 33258 Staining}

After discarding the supernatant, the cells were washed once with PBS, added to 4\% paraformaldehyde and incubated for $10 \mathrm{~min}$. The cells were washed once with PBS, and Hoechst 33258 staining solution was added (Solarbio Bioscience Technology, Beijing, China). The cells were incubated for $5 \mathrm{~min}$ at room temperature in the dark. After aspirating the staining solution, the cells were washed 3 times with PBS for 5 min each and observed under a fluorescence microscope (Olympus, Tokyo, Japan) with 100× magnification. Four fields of view were randomly selected, and the numbers of all cells and apoptotic cells in each field of view were counted to evaluate the percentage of apoptotic cells. (The nuclei of apoptotic cells presented chromatin condensation, marginalization or nuclear beading.)

\section{Apoptosis Rate Detected by Flow Cytometry}

After discarding the supernatant, each group of cells was digested with trypsin. The cells were collected, centrifuged at $1,000 \mathrm{~g}$ for $5 \mathrm{~min}$, washed twice with cold PBS and resuspended in $1 \times$ binding buffer to obtain a $1 \times 10^{6} / \mathrm{mL}$ cell density. Each group of cells $(100 \mu \mathrm{L})$ was transferred into a new centrifuge tube with a pipette, added to $5 \mu \mathrm{L}$ of FITC annexin V and PI (BD Biosciences, San Jose, CA, USA), incubated at room temperature for $15 \mathrm{~min}$ in the dark 


\section{Kidney \\ Blood Pressure \\ Research}

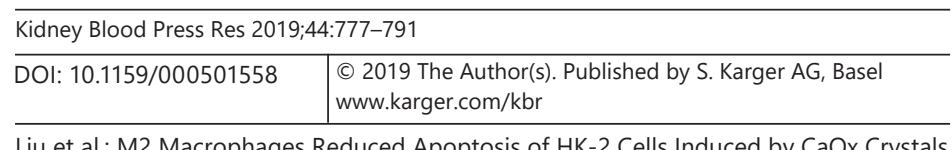

Liu et al.: M2 Macrophages Reduced Apoptosis of HK-2 Cells Induced by CaOx Crystals

and added to $400 \mu \mathrm{L}$ of $1 \times$ binding buffer. The apoptosis rate of each group of cells was measured by flow cytometry (BD Biosciences, San Jose, CA, USA).

\section{ROS Determination}

Each group of cells was digested with trypsin after discarding the supernatant. The cells were collected, centrifuged at $1,000 \mathrm{~g}$ for $5 \mathrm{~min}$ and resuspended in $1.0 \mathrm{~mL}$ of PBS. The cells were then added to $1.0 \mathrm{~mL}$ of $10 \%$ DCFH-DA (Solarbio Bioscience Technology, Beijing, China). The cells were incubated in an incubator for $30 \mathrm{~min}$ and centrifuged at $1,000 \mathrm{~g}$ for $5 \mathrm{~min}$. The supernatant was discarded and the cells were resuspended in $1.0 \mathrm{~mL}$ of PBS. The cells $(200$ $\mu \mathrm{L}$ ) were transferred to a black 96-well plate (Corning, USA) with a micropipette. Fluorescence intensity was immediately detected at a fluorescence wavelength of $488 \mathrm{~nm}$ and an emission wavelength of $525 \mathrm{~nm}$ by using a fluorescence plate reader (Thermo Scientific, USA).

\section{Mitochondrial Membrane Potential Detection}

Each group of cells was digested with trypsin after the supernatant was discarded. The cells were collected, centrifuged at $1,000 \mathrm{~g}$ for $5 \mathrm{~min}$ and resuspended in $1.0 \mathrm{~mL}$ of PBS. The cells were added to $100 \mu \mathrm{L}$ of $2 \mu \mathrm{M}$ TMRE reagent (Cell Signaling Technology, Boston, MA, USA). The cells were incubated in an incubator for $20 \mathrm{~min}$ and centrifuged at $300 \mathrm{~g}$ for $5 \mathrm{~min}$. The supernatant was discarded and the cells were resuspended in $1.0 \mathrm{~mL}$ of PBS. The cells $(100 \mu \mathrm{L})$ were transferred to a black 96-well plate with a micropipette. Fluorescence intensity was immediately detected at a fluorescence wavelength of $550 \mathrm{~nm}$ and an emission wavelength of $580 \mathrm{~nm}$ in a fluorescence plate reader.

\section{Western Blot Analysis}

The protein of the cells was extracted according to the specifications of the manufacturer of RIPA lysate (Beyotime, Shanghai, China). The protein concentration was determined by the BCA (Beyotime, Shanghai, China) method. The protein was added to the SDS loading buffer and boiled at $100{ }^{\circ} \mathrm{C}$ for $5 \mathrm{~min}$. An SDS-polyacrylamide gel was prepared and loaded with $40 \mu \mathrm{g}$ of the protein in each well. Electrophoresis and transfer were performed. A PVDF membrane (Beyotime, Shanghai, China) membrane was blocked with 5\% non-fat dry milk for $1 \mathrm{~h}$. After washing with TBST 3 times, the PVDF membrane was added to solutions of TGF- $\beta$, MCP-1 (Abcam, UK), Akt, phospho-Akt, p47phox, p38, phospho-p38 MAPK, cytochrome c, Bcl-2, cleaved caspase-3 and $\beta$-actin antibodies (Cell Signaling Technology, Boston, MA, USA). After incubation at $4{ }^{\circ} \mathrm{C}$ for $12 \mathrm{~h}$, the membrane was washed 3 times with TBST, added to anti-rabbit IgG $(\mathrm{H}+\mathrm{L})$ antibody (Cell Signaling Technology, Boston, MA, USA) solution and incubated at room temperature for $1 \mathrm{~h}$. After washing the membrane with TBST 3 times, densitometric analysis of protein expression was conducted in an Odyssey system (LI-COR Biosciences, Lincoln, NE, USA).

\section{Statistical Analysis}

Experimental data is expressed as mean \pm SD. SPSS 22.0 statistical software was used for statistical analysis. The mean of multiple groups was compared by one-way analysis of variance. The Tukey method was used for comparison between groups. $p<0.05$ was considered statistically significant.

\section{Results}

Macrophage Polarisation Results

Under the microscope, THP-1 cells appeared round and suspended in the culture medium. M0 macrophages showed inconsistent morphology and adherent growth, and 
A
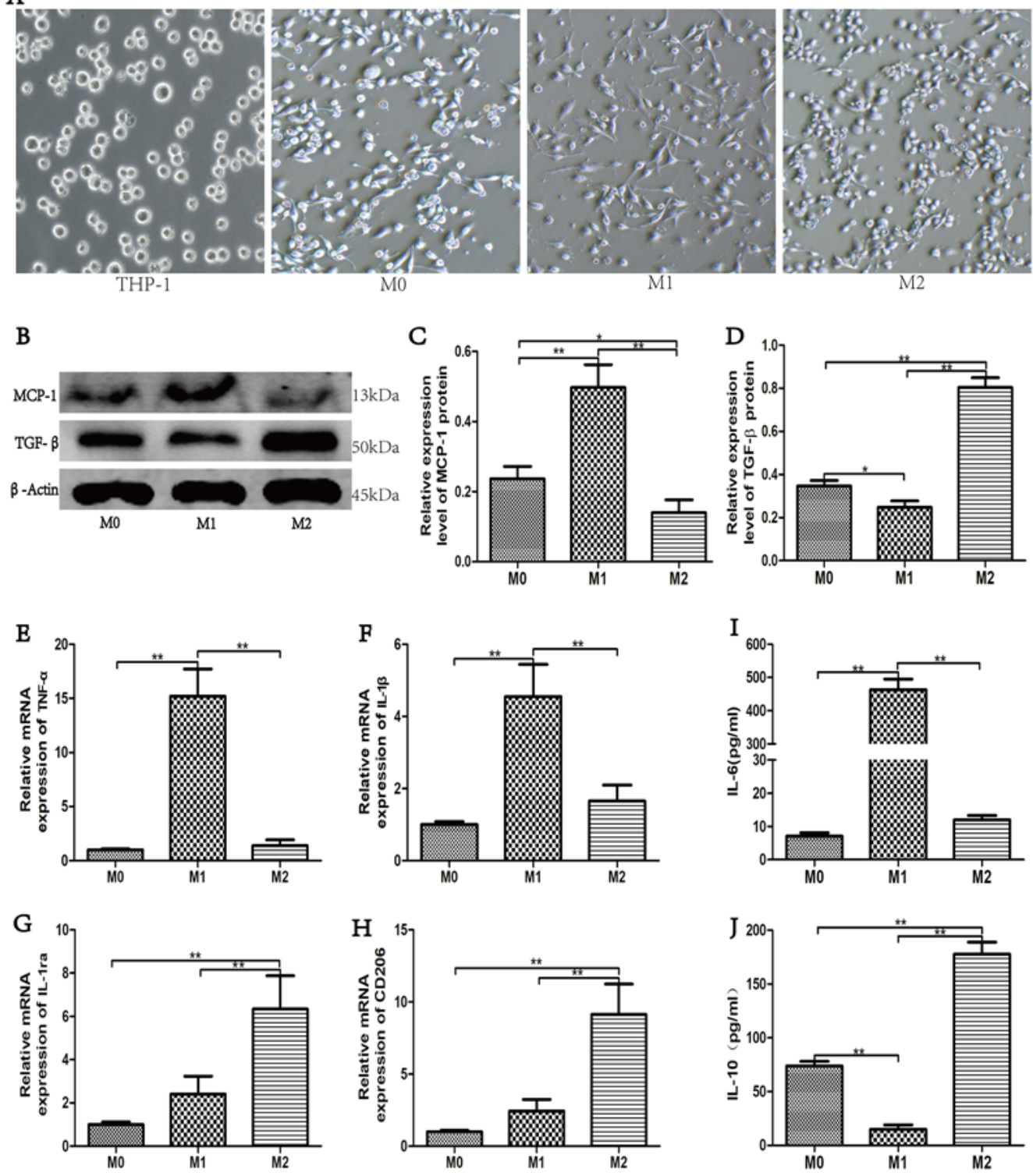

Fig. 1. Polarisation of different types of macrophages. A Morphological observation of the polarised macrophages; (b) representative bands of MCP-1 and TGF- $\beta$ protein by Western blot; (C, D) quantification analysis of MCP-1 and TGF- $\beta$ protein; (E-H) relative mRNA expression levels of TNF- $\alpha$, IL-1 $\beta$, CD206 and IL-1ra detected by reverse transcription-polymerase chain reaction; (I, J) ELISA detection of IL- 6 and IL-10 secretion $\left(^{*} p<0.05,{ }^{* *} p<0.01\right)$. IL, interleukin.

some cells had long antennae. M1 macrophages showed a large number of long antennae and adherent growth. M2 macrophages showed adherent growth, and some cells had a few short antennae (Fig. 1A). The Western blot assay showed that the relative expression of the MCP-1 protein in M1 macrophages was significantly higher than that in M0 and M2 macrophages; moreover, the expression of the TGF- $\beta$ protein in M2 macrophages was significantly higher than that in M0 and M1 macrophages ( $p<0.01$; Fig. 1B-D). The results of reverse tran- 
A

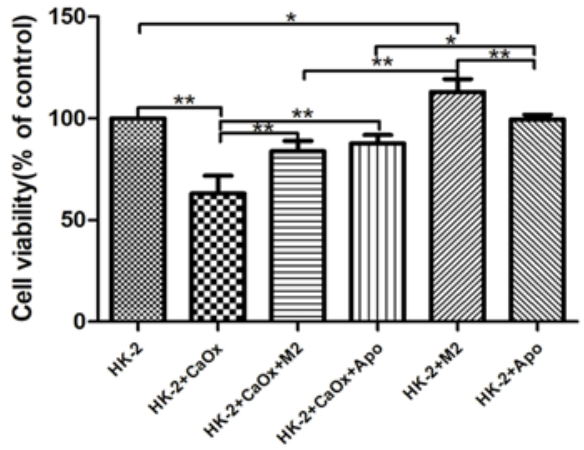

C

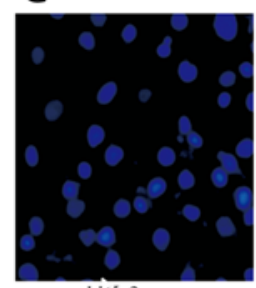

HK-2

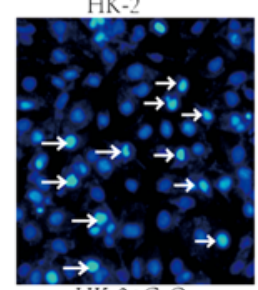

$\mathrm{HK}-2+\mathrm{CaOx}$
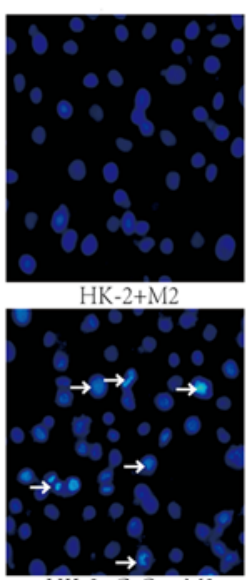

$\mathrm{HK}-2+\mathrm{CaOx}+\mathrm{M} 2$

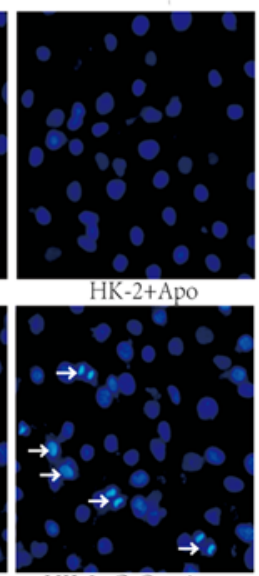

HK $-2+\mathrm{CaOx}+\mathrm{Apo}$
B

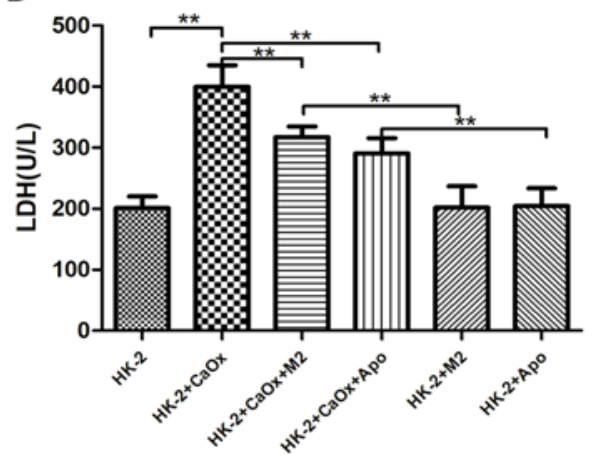

$\mathrm{D}$

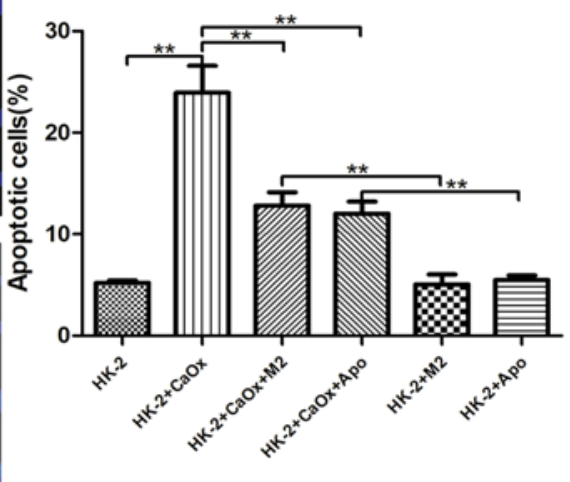

Fig. 2. HK-2 cell damage in each group. A HK-2 cell viability in each group; (B) LDH activity in HK-2 cells in each group; (C) apoptotic staining of Hoechst 33258 cells in each group; and (D) quantitative analysis of CaOx crystals induced apoptosis in each group $\left({ }^{*} p<0.05,{ }^{* *} p<0.01\right)$. CaOx, calcium oxalate; LDH, lactate dehydrogenase.

scription-polymerase chain reaction showed that the relative mRNA expression levels of TNF- $\alpha$ and IL-1 $\beta$ in M1 macrophages were significantly higher than those in M0 and M2 macrophages $(p<0.01$; Fig. 1E, F); the mRNA expression levels of CD206 and IL-1ra in M2 macrophages were significantly higher than those in M0 and M1 macrophages $(p<0.01$; Fig. 1G, H). The results of ELISA showed that the expression of IL-6 in M1 macrophages was significantly higher than that in M0 and M2 macrophages, while the expression of IL-10 in M2 macrophages was significantly higher than that in M0 and M1 macrophages $(p<0.01$; Fig. 1I, J). These results indicated that THP-1 cells were successfully induced to polarise to M1 and M2 macrophages.

\section{Cell Viability}

All experiments were repeated 6 times. The viability of HK-2 cells significantly decreased after stimulation with $\mathrm{CaOx}$ crystals $(p<0.01)$. Under coculture conditions, M2 macrophages significantly promoted the proliferation of HK-2 cells $(p<0.05)$, whereas apocynin had no significant effects $(p<0.05)$. In addition, M2 macrophages and apocynin significantly increased the viability of HK-2 cells treated with $\mathrm{CaOx}$ crystals $(p<0.01$; Fig. $2 \mathrm{~A})$. 


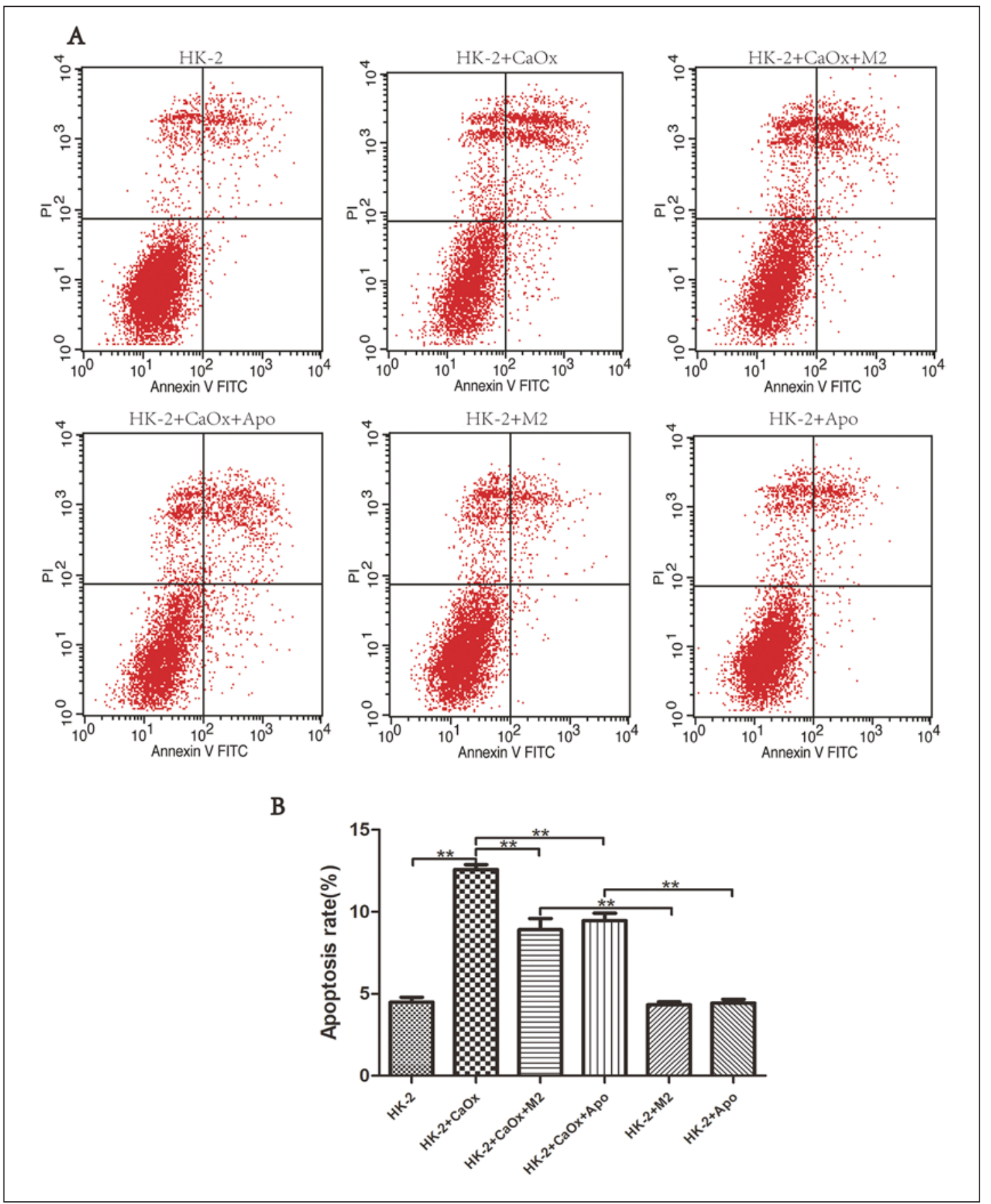

Fig. 3. Apoptosis detected by flow cytometry. A Apoptosis of HK-2 cells in each group determined with flow cytometry; (B) apoptotic rate of HK-2 cells in each group ( $\left.{ }^{* *} p<0.01\right)$. CaOx, calcium oxalate.

\section{LDH Activity of HK-2 Cells in Each Group}

The activity of LDH in HK-2 cells significantly increased after CaOx crystal stimulation $(p<0.01)$, and cell damage was evident. M2 macrophages and apocynin significantly reduced the damage to $\mathrm{HK}-2$ cells after $\mathrm{CaOx}$ crystal stimulation $(p<0.01)$. In addition, M2 macrophages and apocynin exerted no damage on HK-2 cells ( $p<0.05$; Fig. $2 \mathrm{~B})$. 


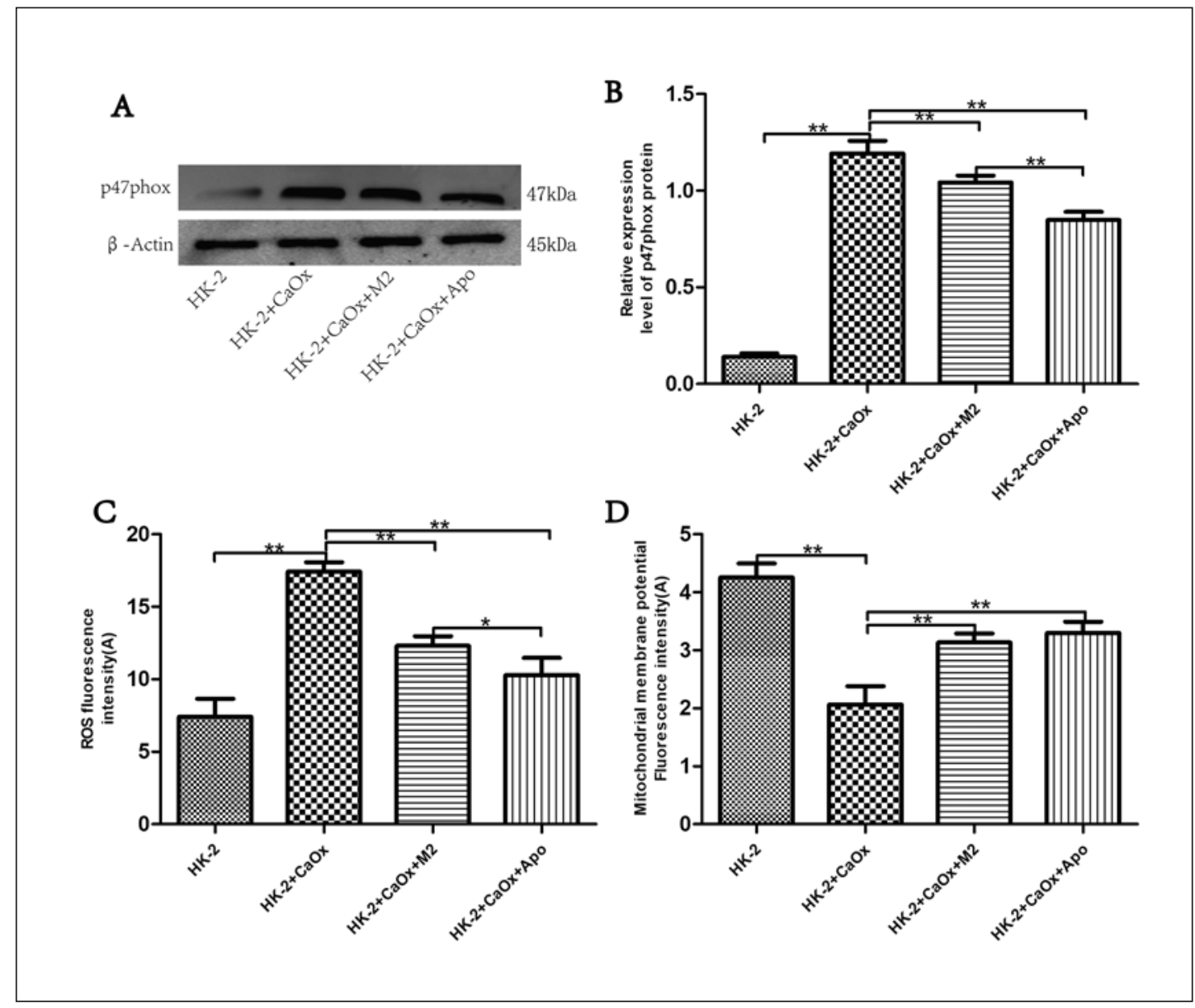

Fig. 4. Oxidative stress damage to HK-2 cells in each group. A Representative bands of p47phox protein in Western blot; (B) quantification analysis of p47phox protein; (C) ROS fluorescence intensity of HK-2 cells in each group; (D) mitochondrial membrane potential fluorescence intensity of HK-2 cells in each group $\left(^{*} p<\right.$ $\left.0.05,{ }^{* *} p<0.01\right)$. CaOx, calcium oxalate; ROS, reactive oxygen species.

\section{Apoptosis Analysis}

The apoptosis of HK-2 cells in each group was analysed by Hoechst 33258 staining and flow cytometry. The cell nuclei in the HK-2 group, HK-2+M2 group and HK-2+Apo group had normal morphology. The cell nuclei in the HK-2+CaOx, HK-2+CaOx+M2 and HK-2+CaOx+Apo groups presented chromatin condensation, marginalization or nuclear beading. However, the number of apoptosing cells in the $\mathrm{HK}-2+\mathrm{CaOx}+\mathrm{M} 2$ and $\mathrm{HK}-2+\mathrm{CaO}+\mathrm{Apo}$ groups was significantly less than that of the HK-2+CaOx group (Fig. 2C, D). The apoptosis rate of HK-2 cells in each group was further examined by flow cytometry; after treatment of HK-2 cells with $\mathrm{CaOx}$ crystals, the apoptotic rate increased significantly $(p<0.01)$, but M2 macrophages and apocynin significantly reduced the apoptotic rate of HK-2 cells after CaOx crystal treatment $(p<0.01$; Fig. 3A, B). The results of Hoechst 33258 staining and flow cytometry showed that M2 macrophages and apocynin reduced the apoptosis of HK-2 cells induced by CaOx crystals.

\section{Western Blot Analysis of p47phox Protein Expression}

After HK-2 cells were treated with CaOx crystals, the protein expression of p47phox significantly increased $(p<0.01)$. After M2 macrophages or apocynin was cocultured with 


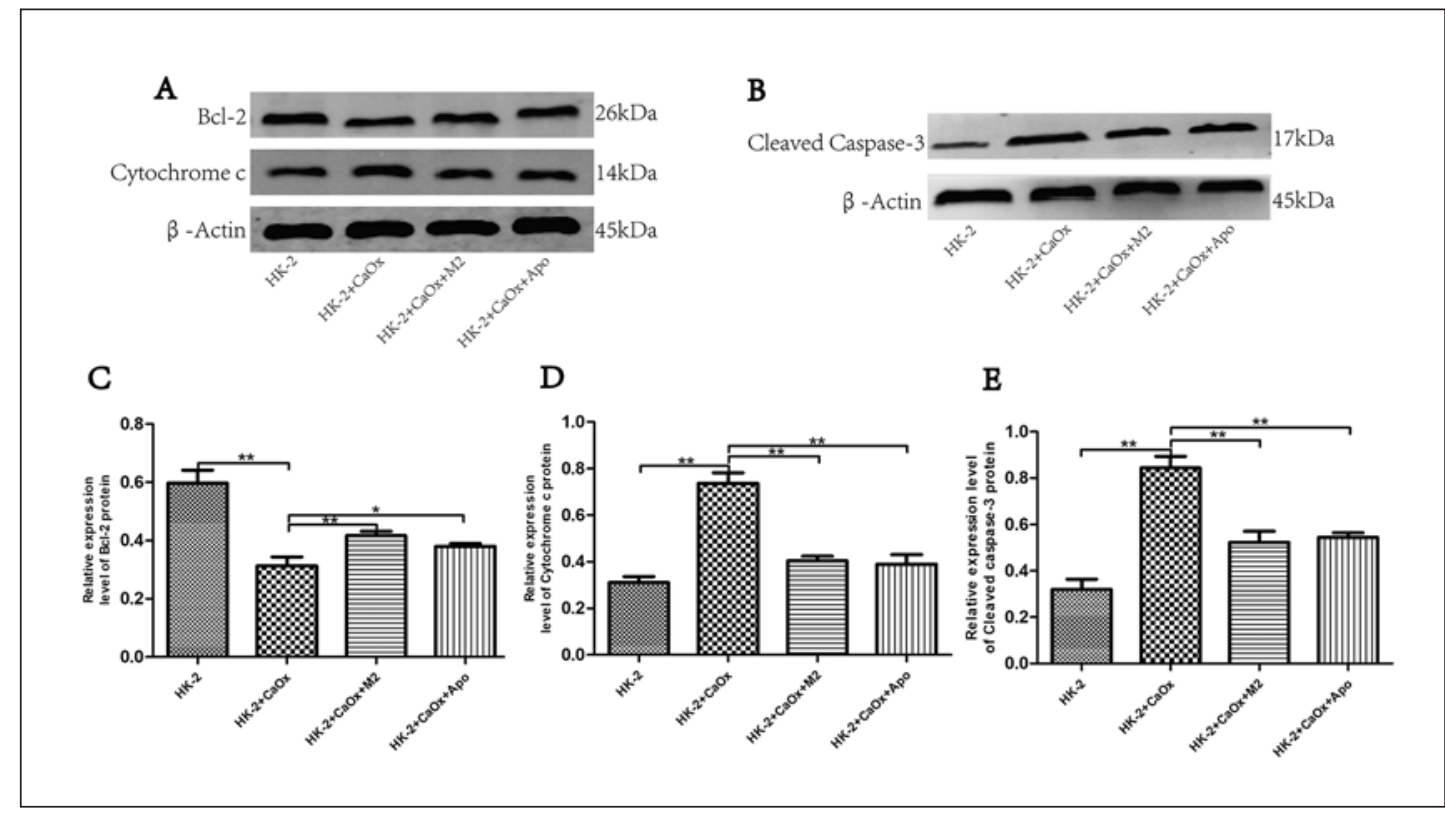

Fig. 5. Expression of apoptosis-related proteins observed by Western blot analysis. A, B Representative bands of Bcl-2, cytochrome c, and cleaved caspase-3 protein by Western blot; (C-E) quantification analysis of Bcl-2, cytochrome $\mathrm{c}$, and cleaved caspase-3 protein $\left({ }^{*} p<0.05,{ }^{* *} p<0.01\right)$. CaOx, calcium oxalate.

HK-2 cells exposed to CaOx crystals, the protein expression of $\mathrm{p} 47$ phox decreased $(p<0.01$; Fig. 4A, B).

\section{ROS Fluorescence Intensity of HK-2 Cells}

The release of ROS increased in HK-2 cells treated with CaOx crystals $(p<0.01)$. The release was reduced by coculturing M2 macrophages or apocynin with HK-2 cells exposed to CaOx crystals $(p<0.01$; Fig. 4 C).

\section{Mitochondrial Membrane Potential}

The mitochondrial membrane potential decreased significantly when HK-2 cells were treated with CaOx crystals. When M2 macrophages or apocynin was cocultured with HK-2 cells exposed to $\mathrm{CaOx}$ crystals, they upregulated the mitochondrial membrane potential $(p<$ 0.01; Fig. 4D).

\section{Expression of Apoptosis-Related Proteins Observed by Western Blot Analysis}

After HK-2 cells were treated with CaOx crystals, the expression of the Bcl-2 protein significantly decreased, whereas the expression of cleaved caspase- 3 and cytochrome c significantly increased $(p<0.01)$. When M2 macrophages or apocynin was cocultured with HK-2 cells exposed to CaOx crystals, the protein expression levels of cytochrome $\mathrm{c}$ and cleaved caspase-3 protein were higher ( $p<0.01$; Fig. $5 \mathrm{~A}-\mathrm{E})$.

\section{Expression of p38 and Akt Detected by Western Blot Analysis}

The expression of $\mathrm{p} 38 \mathrm{MAPK}$ and Akt pathway proteins in HK-2 cells was detected by Western blot analysis. After HK-2 cells were treated with $\mathrm{CaOx}$ crystals, the protein expression of phospho-p38 significantly increased, whereas that of phospho-Akt protein significantly decreased $(p<0.01)$. In addition, M2 macrophages and apocynin downregu- 


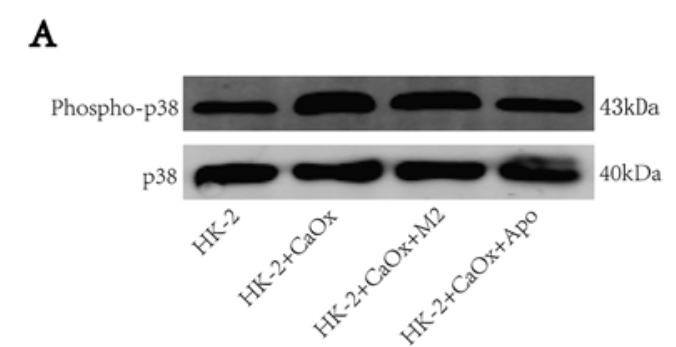

\section{B}
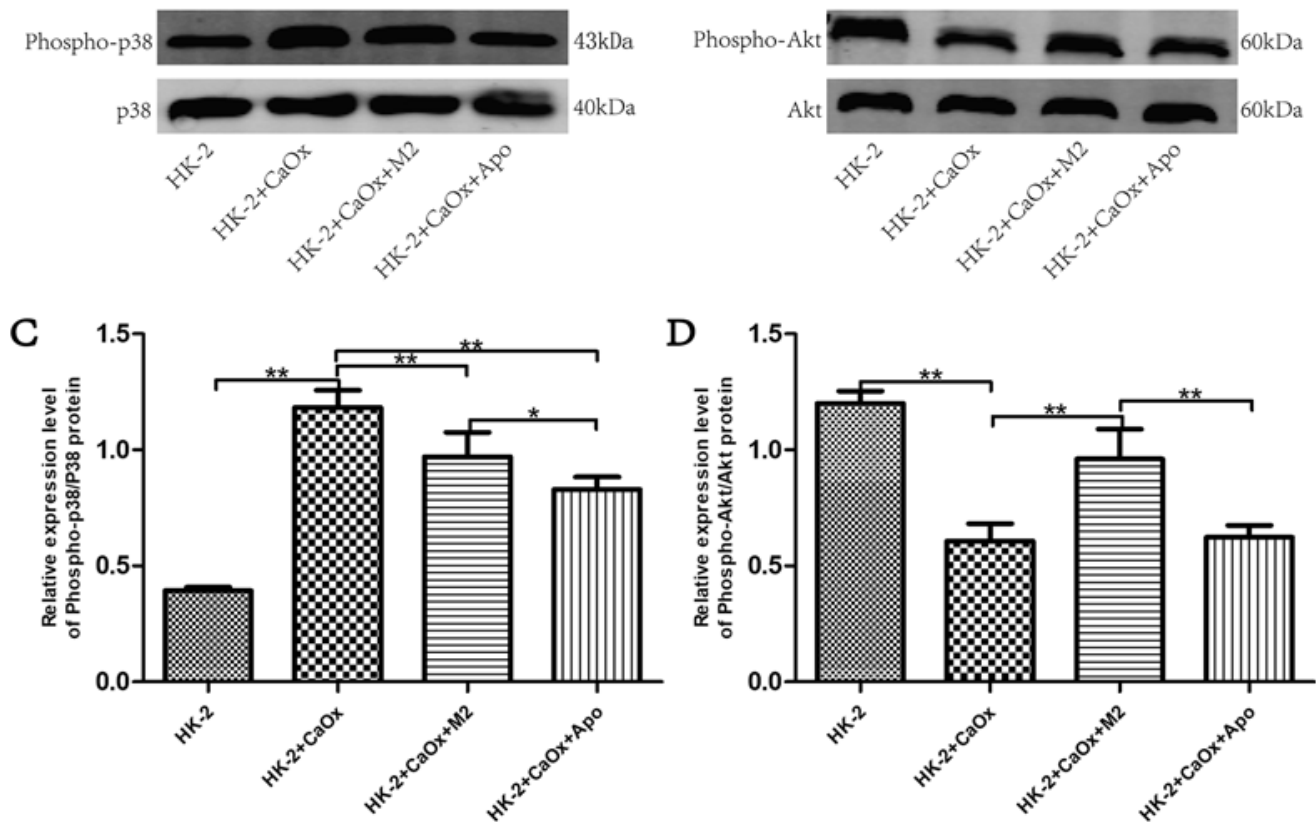

Fig. 6. Western blot analysis of p38 MAPK and Akt protein expression. A, B Representative bands of p38, phospho-p38, Akt, and phospho-Akt protein by Western blot; (C, D) quantification analysis of phosphop38/p38, phospho-Akt/Akt $\left.{ }^{*} p<0.05,{ }^{* *} p<0.01\right)$. CaOx, calcium oxalate.

lated the protein expression of phospho-p38 in HK-2 cells treated with CaOx crystals $(p<$ 0.01). However, M2 macrophages co-cultured with HK-2 cells exposed to CaOx crystals promoted the protein expression of phospho-Akt $(p<0.01)$, but apocynin had no effect $(p<0.05$; Fig. 6A-D).

\section{Discussion}

The factors involved in the formation of kidney stones are extremely complex, and the specific mechanisms remain unclear. At present, methods for preventing the formation of kidney stones are focused on controlling the diet and urine minerals and the use of drugs, such as sodium citrate, thiazine and allopurinol. However, this type of therapy is ineffective in preventing and treating kidney stones and for specific pathologies, such as hyperoxaluria, hypercalciuria, hyperuricaemia and low citric aciduria [23]. Therefore, exploring new ideas for preventing and treating kidney stones has become one of the key research topics in urology.

M1 and M2 macrophages are related to several diseases. The etoposide-mediated apoptosis of human lung adenocarcinoma cells is reduced by M2 macrophages but enhanced by M1 macrophages [24]. In the development of acute kidney injury or chronic kidney disease, M2 macrophages repair kidney damage, whereas M1 macrophages promote renal damage [25]. Taguchi studied the role of M1 and M2 macrophages in the development of kidney stones [11]. The phagocytosis rate of M2 macrophages was found to be higher than that of M1 macrophages. When renal tubular epithelial cells were cocultured with different types of macrophages, M2 macrophages significantly reduced the number of crystals attached. In our 


\section{Kidney \\ Blood Pressure \\ Research}

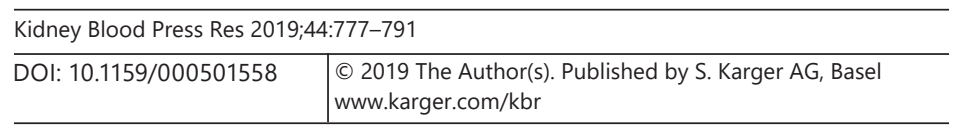

Liu et al.: M2 Macrophages Reduced Apoptosis of HK-2 Cells Induced by CaOx Crystals

previous study, U937 cell-derived M1 macrophages significantly increased inflammatory responses and apoptosis in HK-2 cells induced by hydroxyapatite [26]. However, the mechanism through which M2 macrophages affect the injury and apoptosis of HK-2 cells induced by CaOx crystals remains unclear.

NADPH oxidase consists of 2 membrane-bound subunits, including gp91phox and p22phox, and several cytoplasmic subunits, such as p47phox, p67phox and p40phox [27]. Phosphorylation of the $\mathrm{p} 47 \mathrm{phox}$ subunit plays a key role in regulating NADPH oxidase activity in the kidney, and deposition of kidney stone crystals leads to the activation of angiotensin II [28]. NADPH oxidase is stimulated by activated angiotensin II, and the cytoplasmic subunit p47phox is phosphorylated, leading to ROS production and further damage to kidney cells [29]. Apocynin, an antioxidant and NADPH oxidase inhibitor, blocks the activation of NADPH oxidase by blocking the assembly of cytosolic units and membrane complexes [30, 31]. Damage to tubular epithelial cells induced by oxalate $(\mathrm{Ox})$ and CaOx crystals may be caused by ROS generation after activation of NADPH oxidase [32]. In rats fed apocynin, renal NADPH oxidase $\mathrm{p} 47$ phox activity and $\mathrm{H}_{2} \mathrm{O}_{2}$ release were significantly inhibited, thereby reducing the formation of kidney stones [33]. Therefore, we used apocynin as a positive-control treatment to investigate the specific mechanisms of action of M2 macrophages on CaOx crystal-induced oxidative stress injury and apoptosis of HK-2 cells.

MAPK is a characteristic subfamily of 3 protein kinases, ERK1/2, p38 and JNK. p38 MAPK affects cell growth and death, differentiation, inflammation, oxidative stress and apoptosis [34]. ROS are important intracellular messengers, and ROS signalling seems to trigger the activation of mitochondrial apoptotic pathways by activating p38 [35]. Overproduction of ROS can disrupt mitochondrial membrane potential and activate or inhibit downstream target proteins, including Bcl-2, Bcl-2-associated X, caspase-3 and cytochrome c, thereby regulating various biological functions, including cell growth, proliferation, adhesion and apoptosis [36]. In addition, an important cause of cardiovascular dysfunction is the changed cellular metabolism and apoptosis caused by ROS [37]. The formation of kidney stones has obvious similarities to the formation of vascular calcification [38]. Therefore, the regulation of ROS-related pathways may be an important mechanism for the protection of renal tubular epithelial cells. In the present study, we found that M2 macrophages and apocynin inhibited the injury and apoptosis of HK-2 cells induced by CaOx crystals and downregulated the expression of the NADPH oxidase p47phox protein. Further research found that M2 macrophages and apocynin increased the mitochondrial membrane potential, upregulated the protein expression of the Bcl-2 protein, and inhibited the protein expression of cleaved caspase-3, cytochrome c and phospho-p38 MAPK and the release of ROS in HK-2 cells treated by CaOx crystals. Hence, M2 macrophages and apocynin may reduce the oxidative stress injury and apoptosis of renal tubular epithelial cells treated by CaOx crystals by inhibiting the activation of NAPDH oxidase, reducing the production of ROS and inhibiting the p38 MAPK signalling pathway.

A previous study found that M2 macrophages promoted the proliferation of rat renal tubular epithelial cells [39]. In the present work, M2 macrophages increased the activity of HK- 2 cells, as detected by CCK- 8 assay. M2 macrophages promoted the proliferation of HK-2 cells, whereas apocynin had no effect on proliferation. The PI3K/Akt pathway is an important signalling pathway that promotes cell proliferation, angiogenesis, regulation of metabolic homeostasis [40] and inhibition of apoptosis [41]. ROS produced by oxidative stress can inhibit the PI3K/Akt signalling pathway and directly induce apoptosis [42]. The present experiments found that M2 macrophages activated the phosphorylation of the Akt protein in HK-2 cells treated with CaOx crystals, but apocynin had no effect on it. Hence, M2 macrophages may activate the Akt pathway, promote the proliferation of HK-2 cells and protect against the injury and apoptosis of HK-2 cells treated with CaOx crystals. 


\section{Conclusion}

Our research has revealed that M2 macrophages can protect against the oxidative stress injury and apoptosis of renal tubular epithelial cells induced by CaOx crystals. The mechanism may be through inhibiting the NAPDH oxidase-ROS-p38 MAPK pathway and activating the PI3K/Akt pathway.

M2 macrophages can phagocytose and digest stone crystals; reduce inflammatory reactions, renal tubular epithelial cell damage and apoptosis; and promote the proliferation of damaged cells to prevent the formation of stones. Further studies must determine the relationship between macrophages and kidney stones to provide new ideas for the prevention of the formation of kidney stones by targeting the regulation of M2 macrophage differentiation.

\section{Acknowledgements}

We are grateful to the members of the Clinical Laboratory Center at The First Affiliated Hospital of Guangxi Medical University, Guangxi, People's Republic of China.

\section{Statement of Ethics}

The authors have no ethical conflicts to disclose.

\section{Disclosure Statement}

The authors declare that they have no conflicts of interest to disclose.

\section{Funding Sources}

This study was supported by the National Natural Science Foundation of China (30860280, 30960455, 81360113, and 81760127), Guangxi Province Science Foundation for Youths (No. 2018GXNSFBA138011), and Guangxi Natural Science Foundation (No. 2017GXNSFAA198158).

\section{Author Contributions}

Q.L., Y.L., J.W., Z.H., and J.K. conceived and designed the work that led to the submission, acquired data and played an important role in interpreting the results. X.G. and Z.T. drafted or revised the manuscript. Y.D. approved of the final version.

\section{References}

1 Cheungpasitporn W, Rossetti S, Friend K, Erickson SB, Lieske JC. Treatment effect, adherence, and safety of high fluid intake for the prevention of incident and recurrent kidney stones: a systematic review and metaanalysis. J Nephrol. 2016 Apr;29(2):211-9.

2 López M, Hoppe B. History, epidemiology and regional diversities of urolithiasis. Pediatr Nephrol. 2010 Jan; 25(1):49-59.

3 Romero V, Akpinar H, Assimos DG. Kidney stones:a global picture of prevalence, incidence,and associated risk factors. Rev Urol. 2010;12(2-3):e86-96. 
4 Zisman AL, Evan AP, Coe FL, Worcester EM. Do kidney stone formers have a kidney disease? Kidney Int. 2015 Dec;88(6):1240-9.

5 Wynn TA, Chawla A, Pollard JW. Macrophage biology in development, homeostasis and disease. Nature. 2013 Apr;496(7446):445-55.

6 Murray PJ, Allen JE, Biswas SK, Fisher EA, Gilroy DW, Goerdt S, et al. Macrophage activation and polarization: nomenclature and experimental guidelines. Immunity. 2014 Jul;41(1):14-20.

7 Stöger JL,Gijbels MJ,van der Velden S,Manca M,van der Loos CM,Biessen EA,et al.Distribution of macrophage polarization markers in human atherosclerosis. Atherosclerosis. 2012 Dec;225(2):461-468.

8 Zhang MZ, Wang X, Wang Y, Niu A, Wang S, Zou C, et al. IL-4/IL-13-mediated polarization of renal macrophages/dendritic cells to an M2a phenotype is essential for recovery from acute kidney injury. Kidney Int. 2017 Feb;91(2):375-86.

9 Yang L, Zhang Y. Tumor-associated macrophages: from basic research to clinical application. J Hematol Oncol. 2017 Feb;10(1):58.

10 de Water R, Noordermeer C, Houtsmuller AB, Nigg AL, Stijnen T, Schröder FH, et al. Role of macrophages in nephrolithiasis in rats: an analysis of the renal interstitium. Am J Kidney Dis. 2000 Sep;36(3):615-25.

11 Taguchi K, Okada A, Hamamoto S, Unno R, Moritoki Y, Ando R, et al. M1/M2-macrophage phenotypes regulate renal calcium oxalate crystal development. Sci Rep. 2016 Oct;6:35167.

12 Taguchi K, Okada A, Kitamura H, Yasui T, Naiki T, Hamamoto S, et al. Colony-stimulating factor-1 signaling suppresses renal crystal formation. J Am Soc Nephrol. 2014 Aug;25(8):1680-97.

13 Kusmartsev S, Dominguez-Gutierrez PR, Canales BK, Bird VG, Vieweg J, Khan SR. Calcium Oxalate Stone Fragment and Crystal Phagocytosis by Human Macrophages. J Urol. 2016 Apr;195(4 Pt 1):1143-51.

14 Alelign T, Petros B. Kidney Stone Disease: An Update on Current Concepts. Adv Urol. 2018 Feb;2018:3068365.

15 Khan SR, Pearle MS, Robertson WG, Gambaro G, Canales BK, Doizi S, et al. Kidney stones. Nat Rev Dis Primers. 2017 Jan;3:17001.

16 Liu Y, Li D, He Z, Liu Q, Wu J, Guan X, et al. Inhibition of autophagy-attenuated calcium oxalate crystal-induced renal tubular epithelial cell injury in vivo and in vitro. Oncotarget. 2017 Dec;9(4):4571-82.

17 Umekawa T, Tsuji H, Uemura H, Khan SR. Superoxide from NADPH oxidase as second messenger for the expression of osteopontin and monocyte chemoattractant protein-1 in renal epithelial cells exposed to calcium oxalate crystals. BJU Int. 2009 Jul;104(1):115-20.

18 Khan SR. Reactive oxygen species, inflammation and calcium oxalate nephrolithiasis. Transl Androl Urol. 2014 Sep;3(3):256-76.

19 Wu J, Tao Z, Deng Y, Liu Q, Liu Y, Guan X, et al. Calcifying nanoparticles induce cytotoxicity mediated by ROS-JNK signaling pathways. Urolithiasis. 2019 Apr;47(2):125-35.

20 Kleinman JG, Sorokina EA, Wesson JA. Induction of apoptosis with cisplatin enhances calcium oxalate crystal adherence to inner medullary collecting duct cells. Urol Res. 2010 Apr;38(2):97-104.

21 Liu JJ, Lu Y, Ping NN, Li X, Lin YX, Li CF. Apocynin ameliorates pressure overload-induced cardiac remodeling by inhibiting oxidative stress and apoptosis. Physiol Res. 2017 Nov;66(5):741-52.

22 Kučera J, Binó L, Štefková K, Jaroš J, Vašíček 0, Večeřa J, et al. Apocynin and Diphenyleneiodonium Induce Oxidative Stress and Modulate PI3K/Akt and MAPK/Erk Activity in Mouse Embryonic Stem Cells. Oxid Med Cell Longev. 2016;2016:7409196.

23 Okada A, Yasui T, Fujii Y, Niimi K, Hamamoto S, Hirose M, et al. Renal macrophage migration and crystal phagocytosis via inflammatory-related gene expression during kidney stone formation and elimination in mice: detection by association analysis of stone-related gene expression and microstructural observation. J Bone Miner Res. 2010 Dec;25(12):2701-11.

24 Genin M, Clement F, Fattaccioli A, Raes M, Michiels C. M1 and M2 macrophages derived from THP-1 cells differentially modulate the response of cancer cells to etoposide. BMC Cancer. 2015 Aug;15(1):577.

25 Huen SC, Cantley LG. Macrophages in Renal Injury and Repair. Annu Rev Physiol. 2017 Feb;79(1):449-69.

26 Yu J, Deng Y, Tao Z, Liang W, Guan X, Wu J, et al. The effects of HAP and macrophage cells to the expression of inflammatory factors and apoptosis in HK-2 cells of vitro co-cultured system. Urolithiasis. 2018 Oct;46(5): 429-43.

27 Gill PS, Wilcox CS. NADPH oxidases in the kidney. Antioxid Redox Signal. 2006 Sep-Oct;8(9-10):1597-607.

28 Umekawa T, Hatanaka Y, Kurita T, Khan SR. Effect of angiotensin II receptor blockage on osteopontin expression and calcium oxalate crystal deposition in rat kidneys. J Am Soc Nephrol. 2004 Mar;15(3):635-44.

29 Hanna IR, Taniyama Y, Szöcs K, Rocic P, Griendling KK. NAD(P)H oxidase-derived reactive oxygen species as mediators of angiotensin II signaling. Antioxid Redox Signal. 2002 Dec;4(6):899-914.

30 Lafeber FP, Beukelman CJ, van den Worm E, van Roy JL, Vianen ME, van Roon JA, et al. Apocynin, a plantderived, cartilage-saving drug, might be useful in the treatment of rheumatoid arthritis. Rheumatology (Oxford). 1999 Nov;38(11):1088-93.

31 Touyz RM. Apocynin, NADPH oxidase, and vascular cells: a complex matter. Hypertension. 2008 Feb;51(2): $172-4$.

32 Zuo J, Khan A, Glenton PA, Khan SR. Effect of NADPH oxidase inhibition on the expression of kidney injury molecule and calcium oxalate crystal deposition in hydroxy-L-proline-induced hyperoxaluria in the male Sprague-Dawley rats. Nephrol Dial Transplant. 2011 Jun;26(6):1785-96. 
33 Li CY, Deng YL, Sun BH. Effects of apocynin and losartan treatment on renal oxidative stress in a rat model of calcium oxalate nephrolithiasis. Int Urol Nephrol. 2009 Dec;41(4):823-33.

34 Ono K, Han J. The p38 signal transduction pathway: activation and function. Cell Signal. 2000 Jan;12(1):1-13.

35 Gomez-Lazaro M, Galindo MF, Melero-Fernandez de Mera RM, Fernandez-Gómez FJ, Concannon CG, Segura $\mathrm{MF}$, et al. Reactive oxygen species and p38 mitogen-activated protein kinase activate Bax to induce mitochondrial cytochrome c release and apoptosis in response to malonate. Mol Pharmacol. 2007 Mar;71(3):736-43. Hu L, Sun Y, Hu J. Catalpol inhibits apoptosis in hydrogen peroxide-induced endothelium by activating the PI3K/Akt signaling pathway and modulating expression of Bcl-2 and Bax. Eur J Pharmacol. 2010 Feb;628(13):155-63.

37 Ginter E, Simko V, Panakova V. Antioxidants in health and disease. Bratisl Lek Listy. 2014;115(10):603-6.

38 Khan SR, Rodriguez DE, Gower LB, Monga M. Association of Randall plaque with collagen fibers and membrane vesicles. J Urol. 2012 Mar;187(3):1094-100.

39 Lee S, Huen S, Nishio H, Nishio S, Lee HK, Choi BS, et al. Distinct macrophage phenotypes contribute to kidney injury and repair. J Am Soc Nephrol. 2011 Feb;22(2):317-26.

40 Matthews AT, Ross MK. Oxyradical Stress, Endocannabinoids, and Atherosclerosis. Toxics. 2015;3(4):481-98.

41 Zhang Y, Huang S, Leng Y, Chen X, Liu T, Wang H, et al. Effect of DcR3-specific siRNA on cell growth suppression and apoptosis induction in glioma cells via affecting ERK and AKT. OncoTargets Ther. 2016 Aug;9:5195-202.

42 Cao G, Cai H, Cai B, Tu S. Effect of 5-hydroxymethylfurfural derived from processed Cornus officinalis on the prevention of high glucose-induced oxidative stress in human umbilical vein endothelial cells and its mechanism. Food Chem. 2013 Sep;140(1-2):273-9. 